\title{
Constraint-based Run-time State Migration for Live Modeling
}

\author{
Ulyana Tikhonova \\ Centrum Wiskunde \& Informatica (CWI) \\ Amsterdam, The Netherlands \\ ulyana.tikhonova@cwi.nl \\ Tijs van der Storm \\ Centrum Wiskunde \& Informatica (CWI) \\ Amsterdam, The Netherlands \\ University of Groningen \\ Groningen, The Netherlands \\ t.van.der.storm@cwi.nl
}

\author{
Jouke Stoel \\ Centrum Wiskunde \& Informatica (CWI) \\ Amsterdam, The Netherlands \\ Eindhoven University of Technology \\ Eindhoven, The Netherlands \\ jouke.stoel@cwi.nl \\ Thomas Degueule \\ Centrum Wiskunde \& Informatica (CWI) \\ Amsterdam, The Netherlands \\ thomas.degueule@cwi.nl
}

\begin{abstract}
Live modeling enables modelers to incrementally update models as they are running and get immediate feedback about the impact of their changes. Changes introduced in a model may trigger inconsistencies between the model and its run-time state (e.g., deleting the current state in a statemachine); effectively requiring to migrate the run-time state to comply with the updated model. In this paper, we introduce an approach that enables to automatically migrate such runtime state based on declarative constraints defined by the language designer. We illustrate the approach using NEXTEP, a meta-modeling language for defining invariants and migration constraints on run-time state models. When a model changes, NEXTEP employs model finding techniques, backed by a solver, to automatically infer a new run-time model that satisfies the declared constraints. We apply NeXtep to define migration strategies for two DSLs, and report on its expressiveness and performance.
\end{abstract}

CCS Concepts • Software and its engineering $\rightarrow$ Domain specific languages; Software prototyping; • Theory of computation $\rightarrow$ Programming logic;

\footnotetext{
Permission to make digital or hard copies of all or part of this work for personal or classroom use is granted without fee provided that copies are not made or distributed for profit or commercial advantage and that copies bear this notice and the full citation on the first page. Copyrights for components of this work owned by others than the author(s) must be honored. Abstracting with credit is permitted. To copy otherwise, or republish, to post on servers or to redistribute to lists, requires prior specific permission and/or a fee. Request permissions from permissions@acm.org. SLE '18, November 5-6, 2018, Boston, MA, USA

(C) 2018 Copyright held by the owner/author(s). Publication rights licensed to ACM.

ACM ISBN 978-1-4503-6029-6/18/11_..\$15.00

https://doi.org/10.1145/3276604.3276611
}

Keywords live modeling, run-time state migration, DSL, relational model finding

ACM Reference Format:

Ulyana Tikhonova, Jouke Stoel, Tijs van der Storm, and Thomas Degueule. 2018. Constraint-based Run-time State Migration for Live Modeling. In Proceedings of the 11th ACM SIGPLAN International Conference on Software Language Engineering (SLE '18), November 5-6, 2018, Boston, MA, USA. ACM, New York, NY, USA, 13 pages. https://doi.org/10.1145/3276604.3276611

\section{Introduction}

Live modeling [27, 28] allows users of executable modeling languages to enjoy live, immediate feedback when editing their models, without having to restart execution. Live modeling has its roots in live programming, which allows developers to change program code and use the running application simultaneously, without long edit-compile-restart cycles $[18,21]$. When the programmer changes the program code, its execution state is updated accordingly on-the-fly. This effectively bridges the "gulf of evaluation" [14] between changing a program and the impact of these changes.

While the value of live modeling and live programming is widely recognized [13, 14, 26], engineering live software languages requires a lot of effort and a deep understanding of the host language and its particular domain of application [4].

A central problem for live modeling languages is how to reconcile changes to a model with the run-time state of its execution. Changes to a model or program might invalidate the current run-time state. That is, when the executing model is modified, its run-time state still corresponds to the version before the change. The problem is analogous to migrating a database after a change to the database schema. So the question we address in this paper is how to migrate run-time state after a change to an executing model. 
Earlier work has explored (re)constructing the required migration steps to the run-time state from the changes applied to the model [28]. Unfortunately, this requires intimate operational knowledge of how the change of the model itself is propagated to the runtime in the first place, leading to brittle, imperative, and non-modular code.

In this work, we employ model finding techniques [16] to migrate the run-time state of an executing model. A declarative, constraint-based specification of invariants and migration policies on the run-time state is input to a solver to infer a model that satisfies these constraints, given a representation of the old and new models, and the old run-time state. The resulting model is taken as the new run-time state of the updated version of the DSL program, and execution can continue.

We illustrate this approach using NeXTEP, a prototype meta-modeling language for defining the metamodel of both modeling language and run-time state model, and migration policies as constraints. NEXTEP specifications are then transformed to ALLEAlLE, a language for relational modelfinding modulo theories. The AlLEAlLE specification is then processed by the $\mathrm{Z3}$ [8] constraint solver to obtain a new consistent run-time state instance.

The remainder of this paper is organized as follows. In Section 2, we introduce a motivating example of live modeling and detail the associated challenges. In Section 3, we present a framework for structuring the constraints related to run-time state migration. In Section 4, we introduce NExTEP, our prototype language for live modeling. In Section 5, we evaluate the performance and expressiveness of NeXTEP. Finally, we discuss related work in Section 6 and draw some conclusions in Section 7.

\section{Motivating Example}

Unlike General-Purpose Languages (GPL), DSLs capture the syntax and semantics of a specific domain. As a result, the cognitive distance between DSL models and their dynamic behavior can be arbitrarily large. Live modeling can thus be considered especially beneficial for DSLs because the dynamic effect of a change in a DSL program is harder to predict.

Throughout this paper, we use a simple finite-state machine (FSM) DSL as a running example to explain the ideas and challenges of live modeling, and to illustrate our approach. A program in this DSL is a particular machine that consists of a number of states and transitions. An example scenario of live modeling for the FSM DSL is depicted in Table 1. The starting point (Step \#0) is a simple machine with two states closed and opened and transitions between them. We choose to model the run-time state of machines as the combination of two elements: the current state of execution current and a map visited that keeps track of how many times each state has been visited. Note that the choice of
Table 1. Live modeling scenario 'Add New State' for the FSM DSL

\begin{tabular}{|c|c|c|}
\hline Step & DSL program & Run-time state \\
\hline $\begin{array}{c}\# 0 \\
\text { (initial) }\end{array}$ & opened & $\begin{array}{l}\text { current: } \\
\text { closed } \\
\text { visited: } \\
\text { closed } \mapsto 0 \\
\text { opened } \mapsto 0\end{array}$ \\
\hline $\begin{array}{c}\# 1 \\
\text { (interpreter } \\
\text { step) }\end{array}$ & opened & $\begin{array}{l}\text { current: } \\
\text { opened } \\
\text { visited: } \\
\text { closed } \mapsto 1 \\
\text { opened } \mapsto 0\end{array}$ \\
\hline $\begin{array}{c}\# 2 \\
\text { (user edit) }\end{array}$ & opened & $\begin{array}{c}\text { current: } \\
\text { ??? } \\
\text { visited: } \\
\text { ??? }\end{array}$ \\
\hline
\end{tabular}

what is part of the run-time state and how it is specified is up to the language designer. In particular, it can be specified as an extension of the static metamodel or as a separate semantic domain [6]. Finally, we assume the FSM DSL is executed by a step-based interpreter such that the user can trigger events to trigger transitions, and consequently, modify the run-time state. Changing the model pauses the interpreter for an instant so that the new version can be reloaded.

At Step \#0, the current state is initialized to the initial state closed and every counter is initialized to 0 . At Step \#1, following the first step of execution, the user triggers an event, which changes the current state to opened and increments the visited counter for closed by one. At Step \#2, the user decides to insert a new state locked in the currently running machine. As depicted in Table 1, the question that arises is: what is the new run-time state at this point? Does the newly added state locked become the current state? How should locked be captured in the visited map?

These questions get even more complicated when considering the live modeling scenario depicted in Table 2. In Step \#2 of this scenario, the user chooses to delete the state locked, which is at this point the current state of the machine. Here an obvious question is: which state should be the new current state of the updated machine? Should it be reverted to the initial state of the machine?

A generic live modeling engine cannot provide answers to these questions, because they are inherently domain-specific and should thus comply with domain-specific run-time state migration policies. Ultimately, it is the language designer's 
Table 2. Live modeling scenario 'Remove Current State' for the FSM DSL

$\begin{array}{ll}\text { Step } & \begin{array}{c}\text { Run-time state } \\ \text { current: } \\ \text { closed } \\ \text { visited : } \\ \text { closed } \mapsto 0 \\ \text { opened } \mapsto 0 \\ \text { locked } \mapsto 0\end{array} \\ \text { (interpreter } \\ \text { step) }\end{array}$

responsibility to define these migration policies. We investigate how a language designer can express them in the next section.

\section{Structuring Constraints for Run-time State Migration}

When considering a situation of live modeling, as described in the previous section, we can distinguish two basic components: a static model (denoted $p$ ) and a run-time model (denoted $x$ ). The static model captures the structure (i.e., code) of the DSL program. ${ }^{1}$ The run-time model captures the state of the execution of the DSL program. As we focus on the situation in which a DSL program is changed during its execution, we distinguish two versions of the static and runtime models: $p$ and $x$ before the program has been changed, and $p^{\prime}$ and $x^{\prime}$ after the program has been changed. Thus, we can reformulate the problem of finding a new run-time state as follows: given that $p, x$, and $p^{\prime}$ are known, how do we find $x^{\prime}$ ?

A potential approach would be to modify the model interpreter to repair the run-time state imperatively whenever the program is changed. Unfortunately, as we discuss in more detail in Section 5.3, this approach is error-prone and non-modular. In this paper, we apply model-finding techniques to infer the run-time state based on a set of declarative constraints. Concretely this means identifying requirements

\footnotetext{
${ }^{1}$ Although according to the main idea of live modeling the static model is not static anymore, we still use the term static for the sake of the terminology legacy.
}

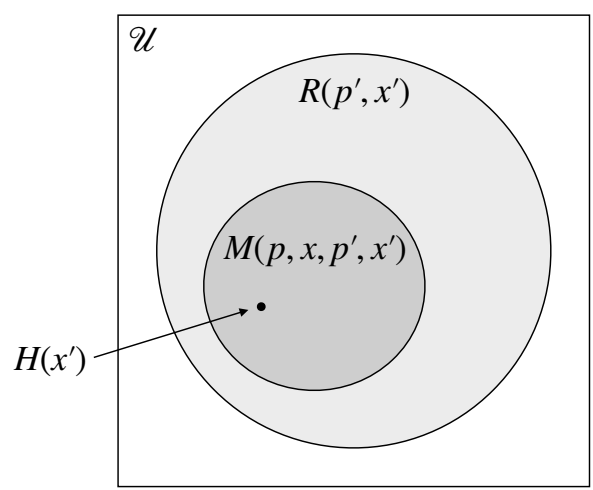

Figure 1. Partitioning and selection of the new run-time model $\left(x^{\prime}\right)$

for $x^{\prime}$ and representing these as a set of constraints in relation to $p, x$, and $p^{\prime}$, and then solving the constraints for $x^{\prime}$. We introduce the following two categories (subsets) of constraints.

Semantic relations $R\left(p^{\prime}, x^{\prime}\right)$ : these constraints ensure that the new run-time state $x^{\prime}$ is semantically consistent with the current (new) version of the DSL model $p^{\prime}$. Note though that there might be multiple-possibly infinite $-x^{\prime}$ that are semantically consistent with $p^{\prime}$.

Migration rules $M\left(p, x, p^{\prime}, x^{\prime}\right)$ : these constraints provide the possibility to take into account what has changed in the DSL model and project this change onto the run-time state. Migration rules restrict the choice of (semantically consistent) $x^{\prime}$ in preference for those $x^{\prime}$ that reflect the change that has happened to the DSL (static) model.

The definition of a solution space by semantic relations $R\left(p^{\prime}, x^{\prime}\right)$ and its filtering by migration rules $M\left(p, x, p^{\prime}, x^{\prime}\right)$ is illustrated by the Venn diagram depicted in Figure 1. Although migration rules restrict the choice of possible solutions, there still can be many (potentially infinite) $x^{\prime}$ that satisfy the semantic constraints. Selecting an arbitrary element of this set as the new run-time state for the updated DSL program might not result in an intuitive new run-time state. For instance, in our previously described FSM scenario 'Add New State' (Table 1), it is perfectly valid to reset the current to opened, and set the visited entities to 1000. Although this would be a correct run-time state (i.e., a valid solution to our set of constraints), it would only add confusion to the user of the DSL.

To find the most suitable new run-time state, we need to introduce a heuristic for selecting one of potentially many solutions. We represent such a heuristic as $H\left(x^{\prime}\right)$ in Figure 1. What are good heuristics may depend on many variables related to domain-specific semantics and usability. One example of a heuristic that we will use in the remainder of this paper is the minimum distance $D\left(x, x^{\prime}\right)$. The minimum 
distance heuristic requires that the new run-time state $x^{\prime}$ should be as close as possible to the previous run-time state $x$. Intuitively, this heuristic captures the principle of "first, do no harm" (i.e., do not break parts of the run-time state that do not have to change), and the "principle of least surprise" (i.e., prefer small incremental changes over invasive ones). In this way, we support the incremental process of editing a DSL model, providing live feedback in relation to the previous run-time state of the DSL program.

To apply constraint-based run-time state migration, language designers must specify the semantic relations $R\left(p^{\prime}, x^{\prime}\right)$ and migration rules $M\left(p, x, p^{\prime}, x^{\prime}\right)$. Semantic relations can be automatically extracted from the DSL definition itself (metamodel, static and dynamic semantics). Migration constraints are explicitly specified to relate $p, p^{\prime}, x$, on the one hand, and $x^{\prime}$, on the other hand.

In the case of the FSM DSL, if the user chooses to delete the state pointed to by current (Table 2), a possible strategy is to reset current to the initial state of the machine. Another strategy is to set the current to the state that was the "closest" to the deleted state. Both policies are equally valid: this is the designer's responsibility to make a choice. In contrast, the heuristic $H\left(x^{\prime}\right)$ is domain-agnostic and can be built into the live modeling tool itself.

Note that our approach infers the new run-time state $\left(x^{\prime}\right)$ only based on the directly preceding step of the execution $(x)$, i.e., migration constraints cannot be defined in terms of the full execution trace. For example, in our FSM DSL we cannot assign the new current to a state that had been current previously in the execution. Taking the execution trace into account is an essential direction for further research.

\section{NeXTEP: a Language for State Migration}

In this section we describe our proof-of-concept implementation of the approach described in Section 3, the NexteP language. In particular, we discuss all described constraints in detail and explain their implementation in NEXTEP for our running example of the FSM DSL.

\subsection{Syntax of NEXTEP}

The NeXtep language allows for formulating preferences for (i.e., for configuring) live modeling in the form of a set of constraints. As described earlier, such constraints are expressed using the constructs of the static and run-time models of a DSL. For this, Nextep uses a simplified version of the Ecore metamodeling language to express classes and references between them. An example Nextep definition for the statemachine DSL is depicted in Listing 1.

A NeXTEP configuration consists of three parts: static, runtime, and migration. The static part (lines 1-10 in Listing 1) describes the static model of the DSL and, in essence, corresponds to (the core of) the DSL metamodel. In our example, it consists of three classes (Machine, State, and

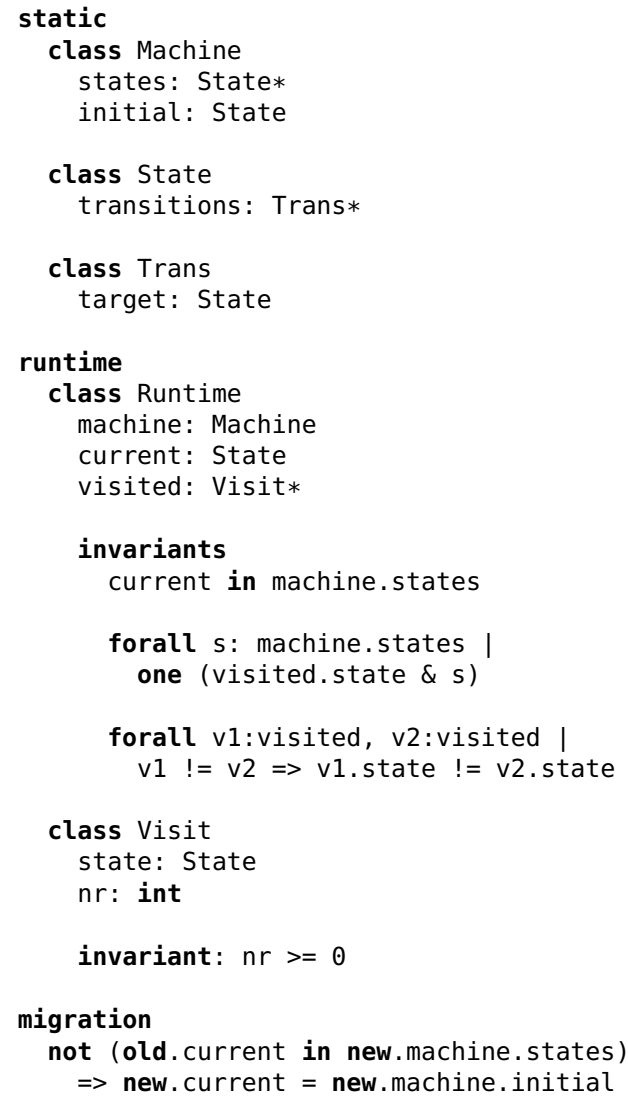

Listing 1. NEXTEP specification of the FSM DSL

Trans) and four references (states, initial, transitions, and target).

References can be of two types: referencing exactly one object (for example, target: State, line 10 in Listing 1) or referencing a set of objects (such as states: State*, line 3 in Listing 1). However, this is not a conceptual limitation of Nextep, but rather a simplification for the sake of demonstration. For the sake of conciseness, we also omit static constraints in the statemachine metamodel from the NEXTEP specification, and assume that these are dealt with by other means, such as parsers or type checkers.

The runtime part of a NEXTEP definition (lines 12-31 in Listing 1) describes the run-time model of the DSL and the semantic relations $R\left(p^{\prime}, x^{\prime}\right)$. The run-time model is defined in the same way as the static model, using classes and references. The semantic relations are defined in the form of invariants attached to these classes.

The class Runtime is a root class that stores references to all components that constitute both the static and the run-time models of the DSL. For the FSM DSL, these are machine for the static model, and current and visited for the run-time model (lines 14-16 in Listing 1). This way, the 
Runtime class defines the scope for the semantic relations $R\left(\mathrm{p}^{\prime}, \mathrm{x}^{\prime}\right)$. We define the following semantic relations for the FSM DSL:

- the current state of the machine is one of its states (line 19 in Listing 1);

- the number of visits is defined exactly once for each state of the statemachine (lines 21-22 in Listing 1);

- different entries in visited correspond to different states of the statemachine, visited is a function (lines 24-25 in Listing 1);

- the number of visits for each state is greater than or equal to zero (line 31 in Listing 1).

The migration part of a Nextep definition (lines 33-35 in Listing 1) describes the migration rules $M\left(p, x, p^{\prime}, x^{\prime}\right)$. Here the keywords old and new denote the instances of the Runtime class corresponding to the old and new versions of the run-time model, respectively. Since the Runtime class refers to the static model, this also provides access to $p$ and $p^{\prime}$. For the FSM DSL, we define the following migration rule: if the current state has been deleted (i.e., not (old current in new.machine.states)), then the new current state is assigned to the initial state (i.e., new. current $=$ new . machine.initial).

\subsection{Semantics of NEXTEP}

In order to implement model finding for our live DSLs, we translate a NEXTEP definition of a DSL and its static and run-time models into AlleAlle. AlleAlle ${ }^{2}$ is a bounded relational model finder. That is, ALLEALLE is a formalism that allows for expressing a model using a combination of first order logic and relational algebra and supports automatic construction of relational instances of such a model in a bounded scope (i.e., limited set of cases) based on the constraints of the model. In addition, AlLEAlle supports declaration of optimization criteria making it suitable for solving optimization problems next to pure satisfiability problems.

As a back-end AlLEAlle uses Z3, an SMT (Satisfiability Modulo Theories) solver [8]. ${ }^{3}$ That is, AlleAlle translates a model expressed in its input language into a corresponding SMT formula and then invokes Z3 to find solution(s) to this formula. The solution is then translated back into an instance of the relational model.

AlLEAlLE is comparable to the Alloy language and analyzer [12]. ${ }^{4}$ The major difference is that, unlike Alloy which is built on top of a SAT solver, ALLEALle is built on top of the Z3 SMT solver, and thus, supports unbounded integer numbers and allows for optimization criteria, which are used by the solver to find the most suitable (optimal) solution.

In this section, we describe the semantics of NexTeP in the form of a translation to AlleAlle. We illustrate our

\footnotetext{
${ }^{2} \mathrm{http}: / /$ github.com/cwi-swat/allealle

${ }^{3} \mathrm{http}: / /$ github.com/Z3Prover/z3

${ }^{4} \mathrm{http}: / /$ alloytools.org
}

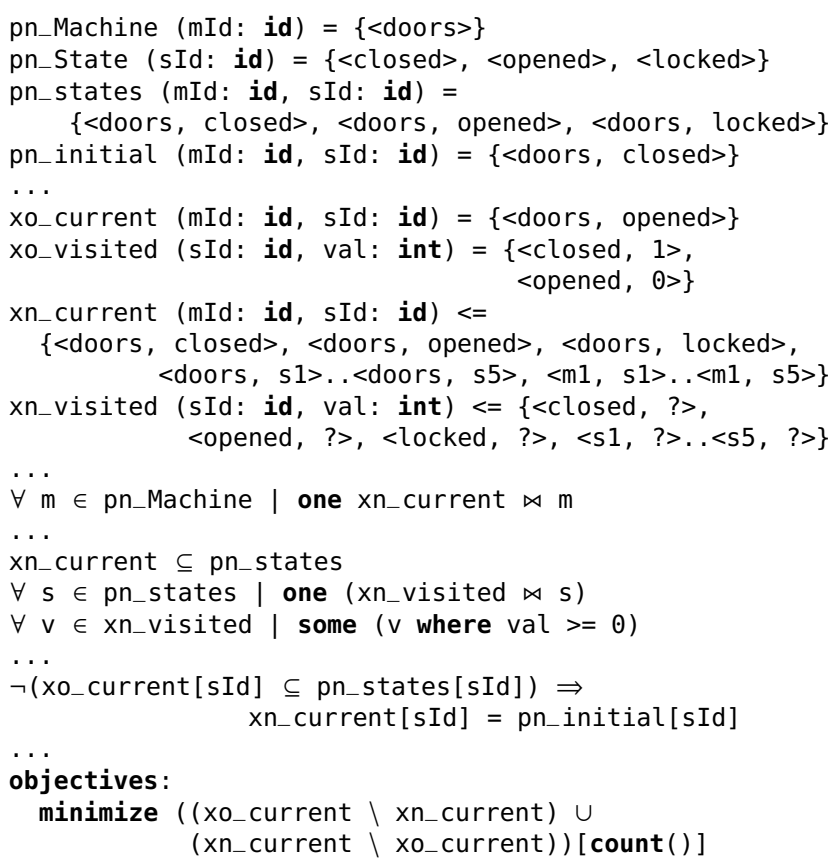

Listing 2. An excerpt of the generated AlleAlle code for the statemachine DSL

description using an excerpt of the AlleAlle specification (see Listing 2) that corresponds to the NeXTEP specification described in the previous section (Listing 1).

Structure translation. All structural elements of both static and dynamic parts of a NeXTEP definition are translated to relations in AlLEAlLE:

- Nextep classes are translated to AlleAlle unary relations;

- Nextep references are translated to AlleAlle binary relations.

As the resulting AlleAlle specification is used for solving our initial problem "given $p, x$, and $p^{\prime}$, what is $x^{\prime}$ ?", all the listed relations are generated for the concrete instances of $p$, $x, p^{\prime}$, and $x^{\prime}$.

For example, in Listing 2 relations with pn as their prefix (lines 1-5) correspond to the new version of the program $p^{\prime}$; relations with $\times 0_{-}$as their prefix (lines 7-8) correspond to the previous run-time state $x$; and relations with $\mathrm{xn}_{-}$as their prefix (lines 10-13) correspond to the new run-time state $x^{\prime}$.

Bounds translation. As mentioned earlier, bounds are used to restrict the scope of search for the back-end solver. In particular, bounds introduce a set of atoms available for the instantiation of relations in an ALLEALLE specification. In the context of NeXTEP, $p, x$, and $p^{\prime}$ are known and, thus, determine the exact bounds for the corresponding AlLEAlle relations. In other words, each of the relations that represent 
the original $p, x$, and $p^{\prime}$ are assigned specific values (i.e., tuples) corresponding to the concrete instances of $p, x$, and $p^{\prime}$ (lines 1-9 in Listing 2, on the right side of the '=' symbol).

While $p, x$, and $p^{\prime}$ are known, the new run-time model $x^{\prime}$ is what we are searching for. Therefore, bounds for the ALLEALLE relations representing $x^{\prime}$ define an extended search scope, not limited to the concrete instances of $p, x$, and $p^{\prime}$. In particular, the $x^{\prime}$ relations are constrained by an upper bound (lines 10-14 in Listing 2, on the right side of the ' $<=$ ' symbol). The upper bounds include the atoms from the instances of $p$, $x$, and $p^{\prime}$, and a finite number of auxiliary atoms of the same type. For instance, in the example, this means that there is one extra machine $\mathrm{m} 1$ and five extra states $\mathrm{s} 1 \ldots \mathrm{s} 5$.

Constraints translation. Semantic relations and migration rules of a NEXTEP definition are translated to the corresponding AlleAlle constraints (formulas) instantiated for $p$, $x, p^{\prime}$, and $x^{\prime}$ (lines 18-24 in Listing 2). Moreover, additional AlleAlle constraints are generated to capture the structural properties of (static and runtime) classes: types and multiplicities of references (line 16 in Listing 2). The expressive power of NexteP constraints is determined by the expressive power of AlLEAlle formulas, which includes relational algebra extended with transitive closure. Thus, NEXTEP supports set theory notation, first-order predicates, (reflexive) transitive closure, and unbounded integer arithmetic. For object navigation we use the standard dot notation.

Minimum distance. As described earlier in Section 3, the heuristic for the minimum distance $D\left(x, x^{\prime}\right)$ is not specific to each particular DSL. In particular, in ALLEAlle, the minimum distance heuristic is generically represented as optimization criteria. For this, $D\left(x, x^{\prime}\right)$ is represented as a set of metrics in AlleAlle, over which the minimization criteria are applied (lines 26-28 in Listing 2).

Minimization is performed over the following metrics:

- the number of elements in the difference of two sets representing $x$ and $x^{\prime}$ correspondingly (such as cur rent in the statemachine DSL);

- the sum of absolute differences between integer values of two vectors representing $x$ and $x^{\prime}$ correspondingly (such as visited in the statemachine DSL).

As a result, for instance, not modifying the current state is preferred over modifying it, because in the former case the set difference will be the empty set, which is minimal. Similarly, minimization of integer values ensures that no arbitrary values will be put in the map visited.

\subsection{Output of NeXTEP}

When applied to the two live modeling scenarios described in Section 2, Nextep produces the following results. Tables 3 and 4 show the instances of $p, x$, and $p^{\prime}$ and the corresponding $x^{\prime}$ for the scenarios of Table 1 and Table 2, respectively.
Here, the instances of $x^{\prime}$ (right bottom corner in both tables) represent the solutions found by the solver. They are constructed by mapping an instance of the relational model (calculated by Z3 and translated to AlLEALlE) on the corresponding instance of the NEXTEP run-time model.

Table 3. Nextep migration for the statemachine scenario 'Add New State،

\begin{tabular}{|c|c|}
\hline $\begin{array}{l}p \\
\text { states: closed, opened } \\
\text { transitions: } \\
\quad(\text { closed } \mapsto \mathrm{t} 1),(\text { opened } \mapsto \mathrm{t} 2) \\
\text { target: } \\
\quad(\mathrm{t} 1 \mapsto \text { opened }),(\mathrm{t} 2 \mapsto \text { closed })\end{array}$ & $\begin{array}{l}x \\
\text { current: opened } \\
\text { visited: } \\
\quad \text { closed } \mapsto 1 \\
\quad \text { opened } \mapsto 0\end{array}$ \\
\hline $\begin{array}{l}p^{\prime} \\
\text { states: closed, opened, locked } \\
\text { transitions: (closed } \mapsto \mathrm{t} 1, \mathrm{t} 3), \\
\quad(\text { opened } \mapsto \mathrm{t} 2),(\text { locked } \mapsto \mathrm{t} 4) \\
\text { target: } \\
\quad(\mathrm{t} 1 \mapsto \text { opened }),(\mathrm{t} 2 \mapsto \text { closed }), \\
\quad(\mathrm{t} 3 \mapsto \text { locked }),(\mathrm{t} 4 \mapsto \text { closed })\end{array}$ & $\begin{array}{l}x^{\prime} \\
\text { current: opened } \\
\text { visited: } \\
\text { closed } \mapsto 1 \\
\text { opened } \mapsto 0 \\
\text { locked } \mapsto 0\end{array}$ \\
\hline
\end{tabular}

Table 4. Nextep migration for the statemachine scenario 'Remove Current State'

\begin{tabular}{|c|c|}
\hline $\begin{array}{l}p \\
\text { states: closed, opened, locked } \\
\text { transitions: (closed } \mapsto \mathrm{t} 1, \mathrm{t} 3), \\
\quad(\text { opened } \mapsto \mathrm{t} 2),(\text { locked } \mapsto \mathrm{t} 4) \\
\text { target: } \\
\quad(\mathrm{t} 1 \mapsto \text { opened }),(\mathrm{t} 2 \mapsto \text { closed }), \\
\quad(\mathrm{t} 3 \mapsto \text { locked }),(\mathrm{t} 4 \mapsto \text { closed })\end{array}$ & $\begin{array}{l}x \\
\text { current: locked } \\
\text { visited: } \\
\text { closed } \mapsto 1 \\
\text { opened } \mapsto 0 \\
\text { locked } \mapsto 0\end{array}$ \\
\hline $\begin{array}{l}p^{\prime} \\
\text { states: closed, opened } \\
\text { transitions: } \\
\quad(\text { closed } \mapsto \mathrm{t} 1),(\text { opened } \mapsto \mathrm{t} 2) \\
\text { target: } \\
\quad(\mathrm{t} 1 \mapsto \text { opened }),(\mathrm{t} 2 \mapsto \text { closed })\end{array}$ & $\begin{array}{l}x^{\prime} \\
\text { current: closed } \\
\text { visited: } \\
\quad \text { closed } \mapsto 1 \\
\quad \text { opened } \mapsto 0\end{array}$ \\
\hline
\end{tabular}

As can be seen in Table 3, the addition of the locked state does not change the runtime state, except for adding a new entry to the visited map, initialized to zero. However, if the current state is removed (i.e., locked in Table 4), the current field is reset to the initial state, and the locked entry is removed from the visited map.

The obtained results are determined by the domain-specific migration policies in the NeXTEP definition (in Listing 1). For example, if we remove the migration rule from our NexteP definition, the calculated $x^{\prime}$ for the scenario 'Remove Current State' would be some arbitrary state (e.g., opened), instead of the initial state of the statemachine. 


\section{Evaluation}

In this section, we use the NeXTEP implementation for an early evaluation of our approach. In Section 5.1, we perform a quantitative evaluation to estimate the performance of NeXteP. In Section 5.2, we perform a qualitative evaluation to challenge the expressive power of Nextep by applying it to a DSL for robotic arm control. Finally, in Section 5.3, we conceptually compare NEXTEP with the manual encoding of migration strategies as presented in previous work [28].

\subsection{Statemachine DSL Case Study}

To evaluate the performance of our method, we ran two different scenarios for the FSM example. ${ }^{5}$

In the first scenario, we tested the live modeling situation where one extra state and two new transitions are added to the new program. The corresponding new run-time state has its visited map extended with an entry for the newly added state assigned to '0' visits. In the second scenario, we tested the case where the current state is removed from the statemachine. This forces the new current to be reset to the initial keeping all the values for the visited map intact, minus the removed state.

In both scenarios, we gradually increased the number of states in the statemachine to assess the impact of model size on performance. The results are shown in Figure 2. The execution times reported in the figure include the following three phases of our implementation: (1) translation of the NexteP specification to the relational specification, (2) translation of the relational specification to SMT constraints (from AlleAlle to SMTLIB), and (3) solving (model finding) by $\mathrm{Z} 3$.

We ran the benchmarks on an early 2015 model MacBook Pro with a $2.7 \mathrm{GHz}$ quad core Intel processor with $8 \mathrm{~GB}$ of DDR3 RAM. We ran each configuration of the benchmark 10 times, ensuring that each execution is independent of the other (i.e., no shared memory, cache, etc.). Figure 2 reports the mean time per configuration of each of the three different phases.

The translation times reported in both scenarios show a similar pattern. The translation of NeXTEP specifications to AlleAlle specifications in the different configurations is fairly stable time-wise with a minimum of $943 \mathrm{~ms}$ and a maximum of $1529 \mathrm{~ms}$.

The translation times from ALLEALLE to SMTLIB also follow a similar trend in both scenarios: the translation time goes up as the NexteP specification grows. An explanation is that increasing the size of NEXTEP specifications causes the number of tuples in the AlleAlle relational models to increase as well. Then, the more tuples in an AlLEAlle specification, the longer it takes to translate from ALLEAlle to

\footnotetext{
${ }^{5}$ The source code of our benchmark experiments can be found at https://github.com/cwi-swat/live-modeling/tree/master/nextstep/src/ benchmark/statemachine
}

SMTLIB. This is especially the case when quantifiers are used in the AlleAlle specifications-and NeXteP relies heavily on these quantifiers.

The solving times reported for Z3 tell a different story. For many configurations, Z3 was not able to find an optimal solution within a time limit of 3 seconds. We empirically set this limit to 3 seconds after observing that Z3 either manages to find a solution within a second or quickly explodes to multiple minutes. Since the time frame of multiple minutes is not in line with the spirit of live modeling, we decided to consider all solving times exceeding 3 seconds as timeouts.

For the first scenario, Z3 timed out for 9 configurations out of 19. For the second scenario, it timed out for 13 configurations out of 19 .

Discussion Our benchmark results suggest that there is much room for improvement in our prototype. These improvements can be classified according to the different phases of our method: improving the translation from NEXTEP to ALLEALLE, improving the translation from ALLEALLE to SMTLIB, and improving the solving times by $\mathrm{Z} 3$.

A first improvement would be to optimize the translation from Nextep to AlleAlle. As mentioned earlier, the current implementation of NEXTEP is an early prototype that has not been aggressively optimized yet. Both the performance of the translation and the generated AlleAlle specifications can be improved. In particular, improving the latter would help in speeding up the total execution time of our method. To verify this intuition, we optimized some AlleAlle specifications manually, bypassing the automatic translation phase of our approach while keeping the exact semantics of the original NexTEP specification, and observed substantial improvements on performance. Generating an optimized ALLEALle specification would help in minimizing the translation time from ALLEALLE to SMTLIB as well, as we have observed that translation times decrease when the $\mathrm{AL}_{\mathrm{L}}$ LEALLE specification shrinks. Finally, generating optimized AlleAlle specifications would also help Z3 to find optimal solutions quicker: the smaller the AllEAlle specification the less variables are declared in the resulting SMT formula, often making it easier for the solver to find optimal solutions.

Next to optimizing NeXTEP, we could look at optimizing AlleAlle. The current implementation of AlleAlle is also in a prototype phase. Early experiments with an optimized native Java version of the AlLEAlle model finder suggest that translation times can potentially be improved by a factor 10 to 15 .

Improving the stability of the solving times of $\mathrm{Z} 3$ is the hardest challenge which for now is considered an open question. As mentioned earlier, generating optimized AlleAlle specifications will help to improve the solving times for Z3. However, whether solving time can be predicted upfront from a given NexTEP specification remains an open question. 

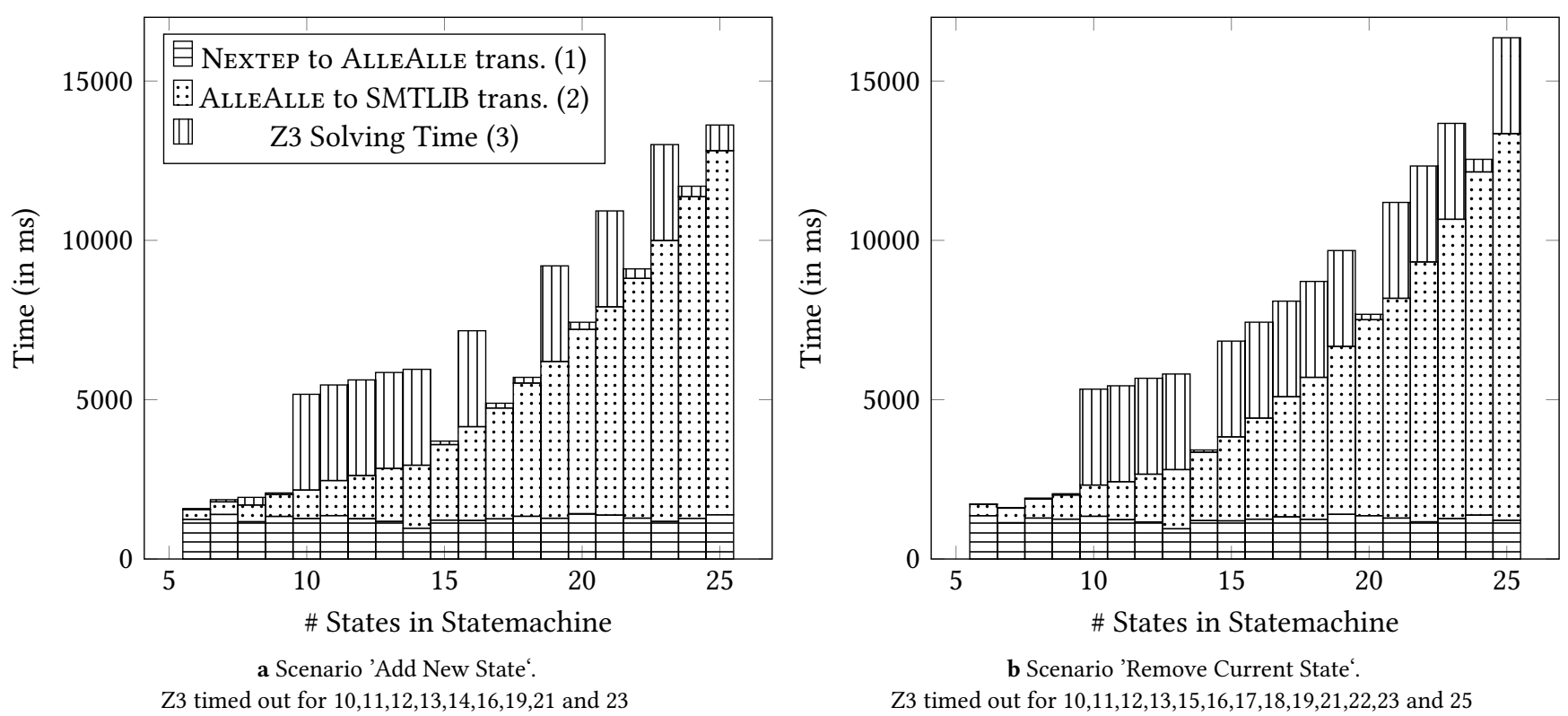

Figure 2. The Statemachine Case Study benchmark results

\subsection{Robotic Arm DSL Case Study}

The Robotic Arm DSL is used for controlling industrial robots that consist of various subsystems (i.e., mechanical and/or electrical parts). For example, a robot can consist of a hand, responsible for manipulating objects, an arm, responsible for moving the hand to a particular position, and smaller subsystems like triggers and actuators. A program in the Robotic Arm DSL describes the coordination of subsystems of a robot during its execution. An example of such a program is shown in the middle column of Table 5.

To perform a certain task, a robot executes specific actions on its subsystems. Each subsystem can execute its actions independently from other subsystems of the robot. However, some of these actions should be executed in certain order, or in other words, some actions have to wait for other actions to be executed first; such dependencies are shown as arrows in Robotic Arm programs. For example, the subsystem hand should not execute the action grab before the subsystem arm has executed the action down (see the DSL program in the second row of Table 5).

The semantics of the Robotic Arm DSL maps a Robotic Arm program to the following execution behavior. In order to execute actions independently, each subsystem maintains a queue of actions that should be executed. First, all actions are collected in one Request pool (Step 0 in Table 5). Then, the actions are taken from the Request one by one and pushed to the corresponding subsystem Queue (Steps 1-2 in Table 5). When an action is executed, it is removed from the queue. To enforce the ordering of actions, an action cannot be queued until all of the actions that this action depends on are in the
Request pool or in the Queues. An exception to this constraint is the situation in which both ordered actions belong to the same subsystem. Then the actions can be queued in the corresponding order. For example, in Step 2 of Table 5, both actions down and up are queued, as they belong to the same subsystem arm.

The bottom row of Table 5 introduces a live modeling step for the Robotic Arm DSL. In particular, interrupting the normal execution of the Robotic Arm program (Steps $0-2$ ), in Step 3 the user updates the program by adding a new subsystem trigger with a new action switch and a new dependency between the actions up and switch. As a result of this update, an obvious migration strategy for the run-time state would be to add a new queue for the subsystem trigger. It is not clear though what to do with the action switch. According to the semantics of the Robotic Arm DSL, the action switch can be executed independently by trigger, and thus, does not need to appear in the Request or Queues. However, intuition suggests that a newly added action should (explicitly) show up in the execution. Therefore, we introduce a migration rule that puts newly added actions to the Request.

The Robotic Arm Nextep definition that captures the described semantic relations and specifies the stated migration rule is depicted in Listing 3. The new run-time state calculated according to this NEXTEP definition is shown in the bottom right cell of Table 5 . Note that in this new run-time state, the newly added action switch is added to the Request and, as a result, the action up is moved (back) to the Request. This allows for observing the execution order according to the newly added dependency. 
Table 5. Update scenario for the Robotic Arm DSL

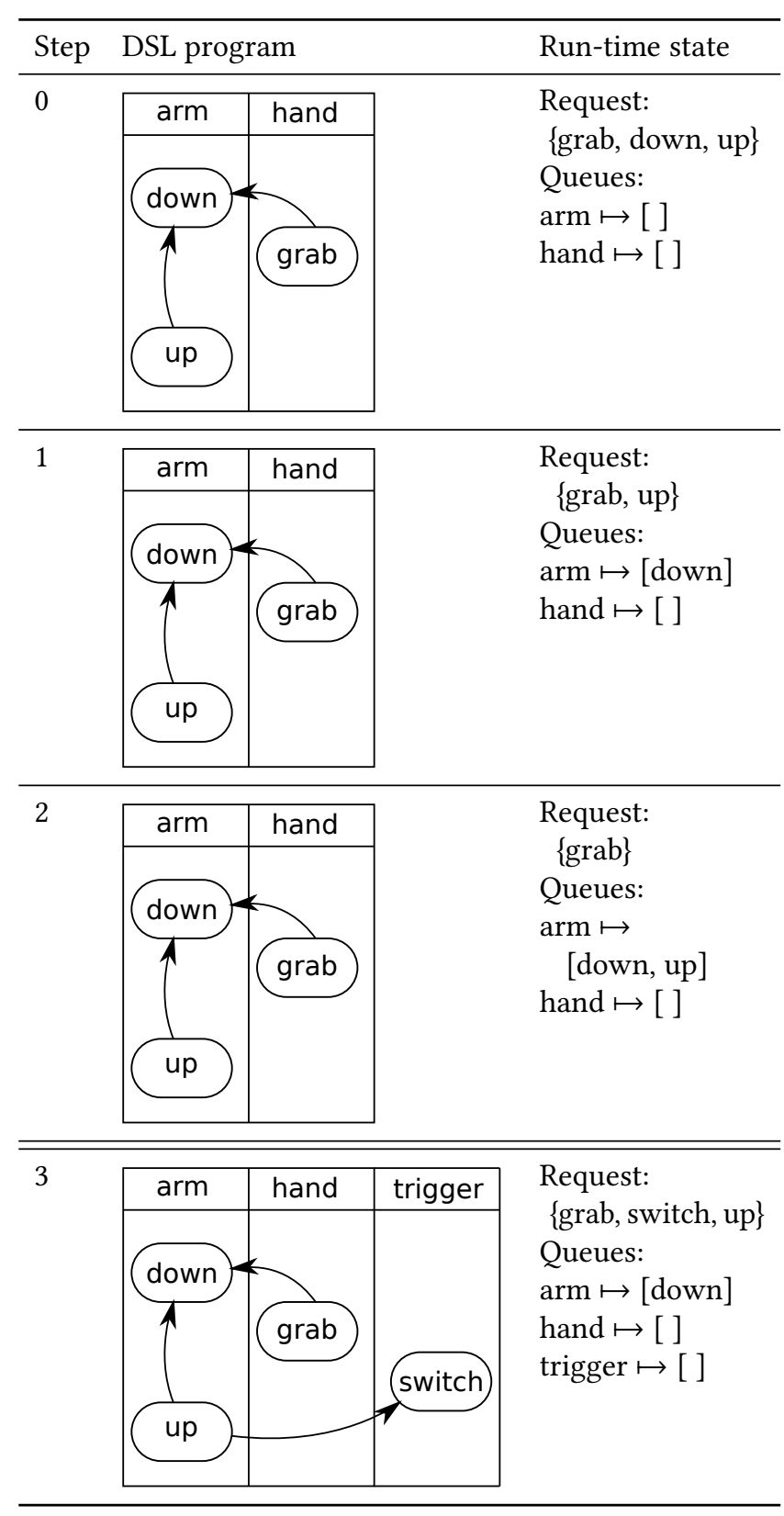

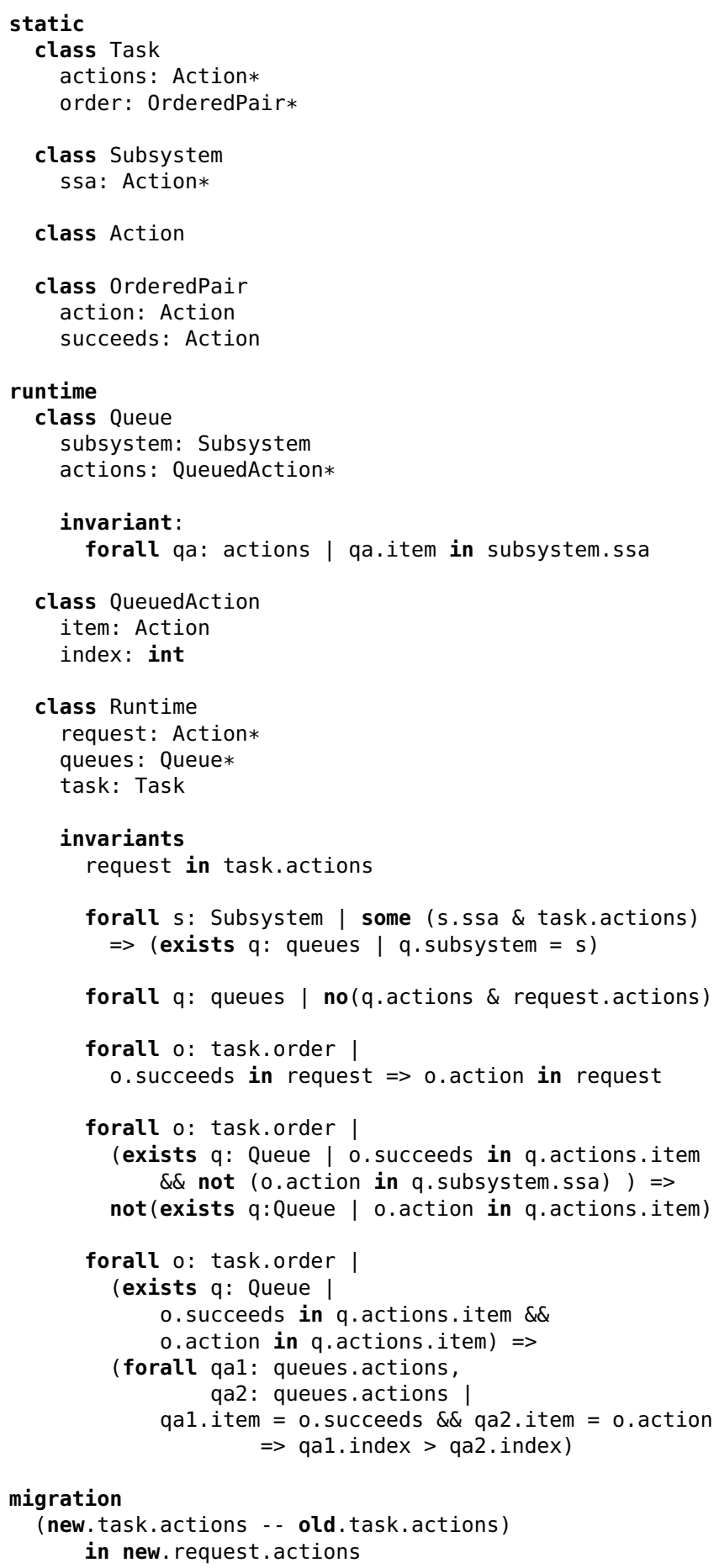

forall q: queues | no(q.actions \& request.actions)

forall o: task.order | 0 . succeeds in request $=>0$.action in request

forall o: task.order | (exists q: Queue | o.succeeds in q.actions.item $\& \&$ not (o.action in q.subsystem.ssa) ) $\Rightarrow$ not(exists q:Queue | o.action in q.actions.item)

forall o: task.order | (exists q: Queue |

0. succeeds in q.actions.item $\& \&$

Listing 3. NexteP definition for the Robotic Arm DSL 
The Nextep definition of Listing 3 defines classes for all constructs introduced above: Task for a Robotic Arm program, Subsystem for a robot subsystem, Action for a subsystem action, OrderedPair to represent order dependencies, Queue and QueuedAction to model a subsystem queue, and Runtime for the run-time state.

The semantics of the Robotic Arm DSL is captured by the following semantic relations:

1. a Queue contains only actions of its corresponding subsystem (line 21);

2. a request contains only actions of the current task (i.e., program being executed) (line 33);

3. if an action of a subsystem appears in the task, then there should be a corresponding queue for this subsystem (lines 35-36);

4. queues and request do not intersect (line 38), i.e., when an action is queued, it is removed from the request;

5. an action, that succeeds another action which is stored in the request, should be also in the request, i.e., should not be queued yet (lines 40-41);

6. an action, that succeeds an action stored in a queue, should not be queued yet, unless these actions belong to the same subsystem (lines 43-46);

7. if two ordered actions belong to the same subsystem queue, then their indexes correspond to the order of the actions (lines 48-55).

Finally, the result that we get for the scenario of Table 5 is to a large extent determined by the migration rule specified in lines 57-59 of Listing 3 according to our earlier design decision. In particular, the constraint states that all actions from the new task (new. task. actions) that are not in the old task (- - old.task.actions) should be added to the request (in new. request.actions).

To conclude, in this section we demonstrated how NexteP can be applied for configuring live modeling for a DSL with a more complicated semantics than our running example of the FSM DSL. The obtained result (i.e., the calculated new run-time state) corresponds to our expectations about the semantic consistency of the DSL and to our intuition about migration strategy of the run-time state.

\subsection{Comparison to Manual Migration}

Earlier work in live modeling explored an operational way of encoding migration strategies, called RMPATCH [28]. RMPAтсH consists of the following steps:

1. after a user changes a DSL program (static model), a delta is computed using a model-based differencing algorithm;

2. a patch corresponding to the delta is then applied to the run-time model, which is a copy of the static model extended with the necessary run-time data;
3. the code that applies the patch to the run-time model is specialized using inheritance and updates the run-time data.

One of the take-aways of the experiment with RMPAтсн is that even for simple examples such as the statemachine language, it is quite hard to correctly implement migrations, and the code quickly becomes unwieldy. Below we consider the main drawbacks of manually coded migrations in contrast with NeXTEP.

Coupling between model and run-time state. RMРАТС requires that run-time state is an instance of the runtime meta-model which is a proper extension of the static metamodel of the language. For instance, the State class defines the number of visits as its own field. Similarly, the class Machine contains the reference to the current state. Using NeXTEP the representation of a run-time state is decoupled from the meta-model, thus allowing for more flexibility for languages where the relation between model and run-time state is less clear cut.

Operational instead of declarative. Second, using program code to encode migration policies requires careful scheduling of operations, since there might be dependencies between modification incurred by the user's model change and migration side effects. In contrast, NExTEP's migration rules are defined as declarative constraints, thus allowing for abstracting away from any ordering constraints.

Hard to reason about correctness. The third problem of encoding migration policies manually using code is that migration is interleaved with the update of (the copy of) the static model within the run-time model. As a result, the run-time model is in an inconsistent state itself when the migrations are being applied. Separating migration logic until after the update has fully finished is non-trivial, since migrations depend on the knowledge of what has changed (i.e., the delta itself).

Scattering of migration logic. Another problem is that migration logic often requires expressing and maintaining global invariants on the run-time state. Modularizing the code according to the type of model elements (e.g., State, Transition, etc.), or delta operations (e.g., Add, Remove, etc.), causes scattering of migration logic over multiple classes and methods.

Lack of extensibility. Finally, as a result of the previous point, migration logic is not modularly extensible. Extending the DSL with new constructs and corresponding additional invariants and rules requires invasive modification of the existing migration code. With NEXTEP, migration rules and invariants can simply be added to the NeXTEP definition, i.e., in conjunction with existing constraints. The potential interactions between constraints are handled by the solver back-end. 
To summarize, Nextep improves upon the earlier migration work in that it provides a declarative, decoupled, and modular way of expressing migration policies. The heavy lifting of finding the new run-time state that is consistent with the new version of the DSL program is delegated to the solver.

\section{Related Work \& Discussion}

In this section, we discuss the related work from three different points of view: the problem of run-time state migration (Section 6.1), the solutions based on constraint solving (Section 6.2), and the area of DSL debugging (Section 6.3). Next to highlighting the existing approaches, we discuss which of their findings we can adopt in our work in order to improve efficiency, expressiveness, and usability of our approach.

\subsection{Model Synchronization}

In this paper, we address the problem of finding a new runtime state that is consistent with an updated DSL program. In a broader perspective, this is a particular case of the problem of keeping different models in sync, which includes well-known sub-problems: consistency checking between different models, change propagation, co-evolution, model repair, etc. As such, this problem has been studied in different research fields and from multiple angles. For instance, the field of views and viewpoints engineering studies the problem of consistency checking between source models and views. The corresponding approaches that address this problem include incremental backward change propagation [23], lenses [11], and triple-graph grammars [22]. Our approach distinguishes itself in letting the language designer specify her own migration policies in a declarative way.

In Model-Driven Engineering (MDE), consistency relations between different models are typically defined through model-to-model transformations. Such model transformations can be used to (re)construct a new (run-time) model for an updated (static) model. Concretely, our work was inspired by the work of Macedo et al. [15, 16], which implements QVT-R bidirectional model transformations using Alloy and its SAT solver to construct consistent models. In comparison, in our work we use ALLEAlLE and the SMT solver as a backend, which allows for more expressiveness when configuring live modeling for a DSL.

Bill et al. use UML and OCL to formalize a synchronization model that includes both a change model and a consistency model [1]. The synchronization model defines inter-model relations and constraints (comparable to our NexteP definition); the change model introduces all possible changes that can be used to construct a new model and assigns a cost to each of such changes. The USE model finder uses these cost values to find the most suitable (optimal) model. On the one hand, defining all possible changes (including languageagnostic, language-specific, and composite changes) requires operational thinking and can result in a tangled and complex model. In our approach, we strive towards a declarative definition. On the other hand, using cost values the authors define five different heuristics for finding the most suitable solution (including the least change strategy that we employ in Nextep). Thus, we plan to leverage their experience in future work to extend NEXTEP with additional heuristics.

\subsection{Constraint-based Solving}

In our approach, we use the SMT solver to find a new runtime model that satisfies the specified constraints. Recently, constraint solvers are being adopted in MDE for the automatic analysis of modeling languages. For instance, Erata et al. present AlloyInEcore, a meta-modeling language that allows for adding Alloy-like invariants into Ecore metamodels [10]. Built on top of the Kodkod (SAT) solver, their tool automatically detects inconsistent models and completes partial models. As both AlloyInEcore and NexteP translate to the relational logic (of Kodkod and AlLEALLE correspondingly), the syntaxes of these two languages are similar. As AlloyInEcore is a more developed and mature language comparing to Nextep, we consider learning from the design decisions of AlloyInEcore in our future work.

Straeten et al. assess the performance of using constraint solvers (Kodkod in particular) for resolving inconsistencies in models [25]. They experiment with models that were reverse-engineered from a set of open-source projects and further translated to Kodkod, to which they add consistency rules formulated directly in Kodkod. The model sizes range from 2064 to 8572 elements. The results obtained in this work demonstrate that "the approach does not provide instantaneous resolution on medium scale models". These results suggest a potential threshold for our approach. However, we believe that we can still significantly improve the performance of NeXTEP.

An alternative approach to employing SAT/SMT solvers is to use constraint programming systems. For instance, Cabot et al. translate UML class diagrams extended with OCL constraints into Constraint Satisfaction Problems (CSPs) and the ECLiPSe constraint programming system to construct model instances [5]. Steimann et al. use constraints embedded into an attribute grammar to check the well-formedness of programs and to compute repair alternatives for malformed programs [24]. Both these works propose various optimizations and search algorithms in order to improve performance of constraint-based model finding.

\subsection{DSL Debugging}

Although the objectives are different, live modeling is also closely related to debugging. Debuggers enable programmers to monitor the execution of a program, set breakpoints, inspect and modify runtime values, and, under certain constraints, hot swap some parts of the code itself. Over time, a 
number of debuggers have been developed for modeling languages, for instance for DEVS [19], fUML [17], or individual diagrams of the Unified Modeling Language (e.g., [7, 9]).

Beyond classical forward-in-time debuggers, Bousse et al. proposed a methodology for the development of generic omniscient debuggers for DSLs [2] backed by efficient and domain-specific execution trace management facilities [3]. Ráth et al. use the VIATRA [29] framework to simulate Petri nets. Users can edit models on-the-fly at simulation time, for instance to resolve cases of non-determinism [20].

We expect many potential cross-fertilization between model debugging and live modeling, and we would like to investigate in the future how our constraint-based approach can solve some of the current problems of debuggers, regarding, for instance, migration of run-time state after code swapping.

\section{Conclusion \& Future Work}

Conclusion Live modeling has the potential to improve the experience of using executable DSLs. Immediate feedback allows DSL users to better assess the impact of the changes they make to their models. A central problem for live modeling languages is how to reconcile changes to a model with the run-time state of its execution. In this paper, we proposed to formulate the problem of run-time state migration in terms of constraints on the run-time state.

We have identified two categories of such constraints: semantic relations which ensure that the new run-time state is consistent with the new version of the DSL model, and domain-specific migration rules which are specified explicitly by the language designer. To choose the most suitable runtime state out of potentially many solutions, we used the heuristic of minimum distance between the new and old run-time states.

We have illustrated this approach using NexTEP, a metamodeling language that allows to define such invariants and migration policies. NEXTEP employs model finding technique, backed by a solver, to automatically infer a new run-time model that satisfies the declared constraints.

We have evaluated the performance of NEXTEP on a simple FSM DSL. Initial results show that performance is satisfactory, albeit unpredictable even for similar problem specifications. Furthermore, we have applied NexTEP to an existing DSL for robotic arm control, which is semantically richer than the FSM DSL. Overall, our results show that constraintbased state migration as realized by NEXTEP is a promising technique for engineering live modeling languages.

Future Work A number of research questions stem from our early prototype and evaluation which we hope to address in future work.

First, we did not address all the steps of the live modeling experience. Once a new run-time state is inferred, how should it be provided back in a seamless way to the user?
How should it be provided to the DSL interpreter itself to let it resume the execution transparently?

Second, we believe user experience plays a crucial aspect in live modeling. How should a user interact with the live modeling framework, through which interface? To be adopted, live modeling must be as close to real time as possible to provide a seamless experience. How to optimize the time it takes to find a new run-time state? We believe that live modeling tools should be evaluated empirically with real users to assess their benefits, and we hope to address these questions in the future.

Finally, we consider the use of a generic minimum distance heuristic to guide the constraint solving process as both a strength and a weakness of our approach. Investigating whether other generic heuristics or domain-specific heuristics could be employed requires more experience with various kinds of DSLs and remains future work.

\section{Acknowledgments}

We would like to thank the anonymous reviewers for their helpful comments. This work is partially supported by the Agile Language Engineering (ALE) associate team (http:// gemoc.org/ale/), and the EU's Horizon 2020 Project No. 732223 CROSSMINER.

\section{References}

[1] Robert Bill, Martin Gogolla, and Manuel Wimmer. 2016. On Leveraging UML/OCL for Model Synchronization. In Proceedings of the 10th Workshop on Models and Evolution co-located with ACM/IEEE 19th International Conference on Model Driven Engineering Languages and Systems (MODELS 2016), Saint-Malo, France, October 2, 2016. (CEUR Workshop Proceedings), Tanja Mayerhofer, Alfonso Pierantonio, Bernhard Schätz, and Dalila Tamzalit (Eds.), Vol. 1706. CEUR-WS.org, 20-29. http://ceur-ws.org/Vol-1706/paper4.pdf

[2] Erwan Bousse, Dorian Leroy, Benoît Combemale, Manuel Wimmer, and Benoit Baudry. 2018. Omniscient debugging for executable DSLs. Journal of Systems and Software 137 (2018), 261-288. https://doi.org/ 10.1016/j.jss.2017.11.025

[3] Erwan Bousse, Tanja Mayerhofer, Benoit Combemale, and Benoit Baudry. 2017. Advanced and efficient execution trace management for executable domain-specific modeling languages. Software \& Systems Modeling (2017), 1-37.

[4] Sebastian Burckhardt, Manuel Fähndrich, Peli de Halleux, Sean McDirmid, Michal Moskal, Nikolai Tillmann, and Jun Kato. 2013. It's alive! continuous feedback in UI programming. In $A C M S I G-$ PLAN Conference on Programming Language Design and Implementation, PLDI '13, Seattle, WA, USA, fune 16-19, 2013. 95-104. https: //doi.org/10.1145/2462156.2462170

[5] Jordi Cabot, Robert Clarisó, and Daniel Riera. 2014. On the verification of UML/OCL class diagrams using constraint programming. fournal of Systems and Software 93 (2014), 1-23. https://doi.org/10.1016/j.jss. 2014.03.023

[6] Benoît Combemale, Xavier Crégut, and Marc Pantel. 2012. A design pattern to build executable DSMLs and associated V\&V tools. In Software Engineering Conference (APSEC), 2012 19th Asia-Pacific, Vol. 1. IEEE, 282-287.

[7] Michelle L. Crane and Jürgen Dingel. 2008. Towards a UML virtual machine: implementing an interpreter for UML 2 actions and activities. In Proceedings of the 2008 conference of the Centre for Advanced Studies 
on Collaborative Research, October 27-30, 2008, Richmond Hill, Ontario, Canada. 8. https://doi.org/10.1145/1463788.1463799

[8] Leonardo de Moura and Nikolaj Bjørner. 2008. Z3: An Efficient SMT Solver. In Tools and Algorithms for the Construction and Analysis of Systems, C. R. Ramakrishnan and Jakob Rehof (Eds.). Springer Berlin Heidelberg, Berlin, Heidelberg, 337-340.

[9] Dolev Dotan and Andrei Kirshin. 2007. Debugging and testing behavioral UML models. In Companion to the 22nd Annual ACM SIGPLAN Conference on Object-Oriented Programming, Systems, Languages, and Applications, OOPSLA 2007, October 21-25, 2007, Montreal, Quebec, Canada. 838-839. https://doi.org/10.1145/1297846.1297915

[10] Ferhat Erata, Arda Goknil, Ivan Kurtev, and Bedir Tekinerdogan. 2018. AlloyInEcore: Embedding of First-Order Relational Logic into MetaObject Facility for Automated Model Reasoning. In Proceedings of the 26th ACM foint European Software Engineering Conference and Symposium on the Foundations of Software Engineering (ESEC/FSE 18). https://doi.org/10.1145/3236024.3264588

[11] J. Nathan Foster, Michael B. Greenwald, Jonathan T. Moore, Benjamin C. Pierce, and Alan Schmitt. 2007. Combinators for bidirectional tree transformations: A linguistic approach to the viewupdate problem. ACM Trans. Program. Lang. Syst. 29, 3 (2007), 17. https://doi.org/10.1145/1232420.1232424

[12] D. Jackson. 2012. Software Abstractions - Logic, Language, and Analysis (revised ed.). MIT press. 336 pages.

[13] Juraj Kubelka, Romain Robbes, and Alexandre Bergel. 2018. The Road to Live Programming: Insights from the Practice. In Proceedings of the 40th International Conference on Software Engineering (ICSE '18). ACM, New York, NY, USA, 1090-1101. https://doi.org/10.1145/3180155. 3180200

[14] Henry Lieberman and Christopher Fry. 1995. Bridging the gulf between code and behavior in programming. In Proceedings of the SIGCHI conference on Human factors in computing systems. ACM Press/AddisonWesley Publishing Co., 480-486.

[15] Nuno Macedo and Alcino Cunha. 2013. Implementing QVT-R Bidirectional Model Transformations Using Alloy. In Fundamental Approaches to Software Engineering - 16th International Conference, FASE 2013, Held as Part of the European foint Conferences on Theory and Practice of Software, ETAPS 2013, Rome, Italy, March 16-24, 2013. Proceedings. 297-311. https://doi.org/10.1007/978-3-642-37057-1_22

[16] Nuno Macedo, Tiago Guimarães, and Alcino Cunha. 2013. Model repair and transformation with Echo. In 2013 28th IEEE/ACM International Conference on Automated Software Engineering, ASE 2013, Silicon Valley, CA, USA, November 11-15, 2013, Ewen Denney, Tevfik Bultan, and Andreas Zeller (Eds.). IEEE, 694-697. https://doi.org/10.1109/ASE. 2013.6693135

[17] Tanja Mayerhofer, Philip Langer, and Gerti Kappel. 2012. A runtime model for fUML. In Proceedings of the 7th Workshop on Models@ run. time. ACM, 53-58.

[18] Sean McDirmid. 2013. Usable live programming. In ACM Symposium on New Ideas in Programming and Reflections on Software, Onward!
2013, part of SPLASH '13, Indianapolis, IN, USA, October 26-31, 2013. 53-62. https://doi.org/10.1145/2509578.2509585

[19] Simon Van Mierlo, Yentl Van Tendeloo, and Hans Vangheluwe. 2017. Debugging Parallel DEVS. Simulation 93, 4 (2017), 285-306. https: //doi.org/10.1177/0037549716658360

[20] István Ráth, David Vago, and Dániel Varró. 2008. Design-time simulation of domain-specific models by incremental pattern matching. In IEEE Symposium on Visual Languages and Human-Centric Computing, VL/HCC 2008, Herrsching am Ammersee, Germany, 15-19 September 2008, Proceedings. 219-222. https://doi.org/10.1109/VLHCC.2008.4639089

[21] Patrick Rein, Stefan Ramson, Jens Lincke, Robert Hirschfeld, and Tobias Pape. 2019. Exploratory and Live, Programming and Coding - A Literature Study Comparing Perspectives on Liveness. Programming fournal 3, 1 (2019), 1. https://doi.org/10.22152/programming-journal. $\operatorname{org} / 2019 / 3 / 1$

[22] Andy Schürr. 1994. Specification of Graph Translators with Triple Graph Grammars. In Graph-Theoretic Concepts in Computer Science, 20th International Workshop, WG '94, Herrsching, Germany, fune 16-18, 1994, Proceedings. 151-163. https://doi.org/10.1007/3-540-59071-4_45

[23] Oszkár Semeráth, Csaba Debreceni, Ákos Horváth, and Dániel Varró. 2016. Incremental backward change propagation of view models by logic solvers. In Proceedings of the ACM/IEEE 19th International Conference on Model Driven Engineering Languages and Systems, SaintMalo, France, October 2-7, 2016. 306-316. http://dl.acm.org/citation. cfm?id $=2976788$

[24] Friedrich Steimann, Jörg Hagemann, and Bastian Ulke. 2016. Computing Repair Alternatives for Malformed Programs Using Constraint Attribute Grammars. In Proceedings of the 2016 ACM SIGPLAN International Conference on Object-Oriented Programming, Systems, Languages, and Applications (OOPSLA 2016). ACM, New York, NY, USA, 711-730. https://doi.org/10.1145/2983990.2984007

[25] Ragnhild Van Der Straeten, Jorge Pinna Puissant, and Tom Mens. 2011. Assessing the Kodkod Model Finder for Resolving Model Inconsistencies. In Modelling Foundations and Applications - 7th European Conference, ECMFA 2011, Birmingham, UK, fune 6- 9, 2011 Proceedings (Lecture Notes in Computer Science), Robert B. France, Jochen Malte Küster, Behzad Bordbar, and Richard F. Paige (Eds.), Vol. 6698. Springer, 69-84. https://doi.org/10.1007/978-3-642-21470-7_6

[26] Steven L. Tanimoto. 2013. A perspective on the evolution of live programming. In Proceedings of the 1st International Workshop on Live Programming, LIVE 2013, San Francisco, California, USA, May 19, 2013, Brian Burg, Adrian Kuhn, and Chris Parnin (Eds.). IEEE Computer Society, 31-34. https://doi.org/10.1109/LIVE.2013.6617346

[27] Tijs van der Storm. 2013. Semantic deltas for live DSL environments. In 1st International Workshop on Live Programming (LIVE). IEEE, 35-38.

[28] Riemer van Rozen and Tijs van der Storm. 2017. Toward live domainspecific languages. Software \& Systems Modeling (14 Aug 2017). https: //doi.org/10.1007/s10270-017-0608-7

[29] Dániel Varró and András Balogh. 2007. The model transformation language of the VIATRA2 framework. Sci. Comput. Program. 68, 3 (2007), 214-234. https://doi.org/10.1016/j.scico.2007.05.004 